\title{
Energy Storage System Control for Energy Management in Advanced Aeronautic Applications
}

\author{
A. Cavallo, G. Canciello, and B. Guida \\ Department of Industrial and Information Engineering, Second University of Napoli, 81031 Aversa, Italy \\ Correspondence should be addressed to A. Cavallo; alberto.cavallo@unina2.it
}

Received 2 December 2016; Accepted 5 April 2017; Published 19 April 2017

Academic Editor: Ilhan Tuzcu

Copyright (c) 2017 A. Cavallo et al. This is an open access article distributed under the Creative Commons Attribution License, which permits unrestricted use, distribution, and reproduction in any medium, provided the original work is properly cited.

\begin{abstract}
In this paper an issue related to electric energy management on board an aircraft is considered. A battery pack is connected to a high-voltage bus through a controlled Battery Charge/Discharge Unit (BCDU) that makes the overall behaviour of the battery "intelligent." Specifically, when the aeronautic generator feeding the high-voltage bus has enough energy the battery is kept under charge, while if more loads are connected to the bus, so that the overload capacity of the generator is exceeded, the battery "helps" the generator by releasing its stored energy. The core of the application is a robust, supervised control strategy for the BCDU that automatically reverts the flow of power in the battery, when needed. Robustness is guaranteed by a lowlevel high gain control strategy. Switching from full-charge mode (i.e., when the battery absorbs power from the generator) to generator mode (i.e., when the battery pumps energy on the high-voltage bus) is imposed by a high-level supervisor. Different from previous approaches, mathematical proofs of stability are given for the controlled system. A switching implementation using a finite-time convergent controller is also proposed. The effectiveness of the proposed strategy is shown by detailed simulations in Matlab/Stateflow/SimPowerSystem.
\end{abstract}

\section{Introduction}

At the beginning of the new millennium, in a pioneering paper [1] the authors presented a set of ideas and visions for the aircraft of the future. Great emphasis was given to possible replacement of different actuators with electric ones (e.g., basically electric motors with different technologies). Moreover, innovative, "smart" materials are investigated for noise and vibration reduction onboard $[2,3]$, and the key point is the possibility of converting electric energy into mechanical motion. It is clear that the increased use of electric devices implies new concepts for power generation, distribution, and management of the electric energy onboard [4]. Nowadays "More Electric Aircraft (MEA)" has become a keyword in the design of next generation aircraft. The advantages of MEA are apparent in terms of increased fault-tolerance capabilities and reduction of pollution resulting from possible weight reduction. As a consequence, many research projects are currently funded world-wide on this topic. For instance, in Europe several Framework Programmes [5-8] or the more recent initiatives CleanSky and CleanSky2 [9] have a large number of projects oriented to the MEA. However, it is clear that the expanded request of energy on board has to be traded off against the requirement to keep size and weight at acceptable levels. Thus, there is a need for rethinking traditional electric devices onboard, or at least to optimise their usage.

Basically, all the aircraft power grids employ at least two DC power busses. The main bus is a high-voltage (HV) bus that is powered by a three-phase electric generator, followed by a rectification stage, for example, via an Autotransformer Rectifier Unit (ATRU). The typical value of DC voltage on the $\mathrm{HV}$ bus is $270 \mathrm{~V}$. The second bus is a low-voltage (LV) bus that is supplied by one or more batteries at $28 \mathrm{~V}$. The HV bus supplies the "heavy" loads onboard, for example, icing protection system, air conditioning, and cabin pressurisation, while the battery acts at engine start-up, supplies the avionic loads, and is called for in exceptional cases, as in the case of fault of the electric generator. Usually, energy is transferred from the HV bus to the LV one (e.g., in order to charge the battery) by using a buck converter, while a boost converter permits the battery to power the starter-generator. Obviously, 
the idea to allow the flow of energy in both directions (Highto-Low and Low-to-High) requires a bidirectional converter or to use two unidirectional converters antiparallel, which increases the weight, cost, and space. For these reasons, we will refer to the bidirectional solution. One of the main advantages of using the batteries onboard to supply power to the loads is the possibility of reducing the weight of the electric generator, by reducing its size. It is well known [10] that electric generator sizing is based on the so-called 5 seconds' and 5 minutes' overload capability that are a piecewise linear approximation of the true overload curve of the generator. Roughly speaking, the generator is assumed to be able to withstand a large overload for the first 5 seconds, while the 5 minutes' overload level $P_{\mathrm{OL}}$ simply indicates a maximum level that the generator can supply in steady-state. Obviously, if a total load is connected to the generator such that, after a transient of maximum duration $5 \mathrm{~s}$, it requires more power than the rated $P_{\mathrm{OL}}$, generator sizing has to be increased. In other words, if after $5 \mathrm{~s}$ a power level below $P_{\mathrm{OL}}$ was guaranteed, no oversized related to 5 minutes capabilities would be needed, thus reducing size and weight of the generator. This is exactly the point where the battery can be called for: the objective is to use the battery to supply energy to the HV bus, thus helping the generator [11] in the case of overload. Applications of this approach are found in the aeronautical field [12] or in automotive applications, with the increased interest for hybrid vehicles [13].

From this perspective, the key device for energy management of the battery is a bidirectional converter. Since power converters are usually switching devices, their natural control strategy turns out to be sliding mode control [14]. Moreover, in order to keep the commutation frequency fixed, PWM implementation has also been proposed adopting a $\Sigma \Delta$ modulation [15]. Different sliding-based controllers have been proposed for any DC/DC converter type. A typical approach is an indirect control, with a double nested loop [16], that is, an inner current loop and an outer voltage loop. However, in [17] it has been shown that, by using a suitable converter topology and second-order sliding manifold concepts, also direct voltage control can be achieved. The next problem is to be able to guarantee the stability of the controlled converter when reversing the direction of the current. This problem has been addressed recently for Buck-Boost converters in [18], where an estimate of the region of attraction for each control mode (buck and boost) was provided.

In this paper a different approach to converter control is adopted, again considering the topology in [17], but focusing on current control in the converter's inductor. Specifically, only two phases are considered in the working condition of the converter, namely, a standard operation phase, when the generator has power enough to recharge the battery, and a generator overload phase, in which the controller may reduce the charge to the battery, or even reverse the flow of energy (from the battery to the loads on the HV bus) in order to overcome the overload. A suitable selection of a sliding manifold where the controlled system state is forced to evolve makes it easy to check the stability of the closedloop system that becomes linear. This simplifies also the

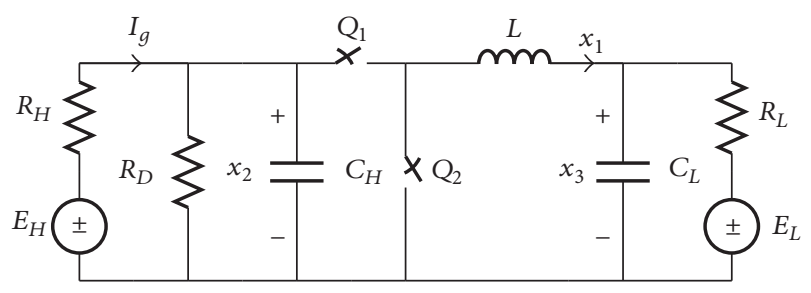

FIGURE 1: Bidirectional converter schematic.

stability analysis for the overall design. Finally, also a finitetime implementation of the control is taken into account [19].

A simulation scenario considering a normal operating condition, with two different load conditions, followed by two different overloads (the second being more severe than the first) is presented, showing the effectiveness and robustness of the proposed strategy.

\section{BBCU Model}

The topology of the bidirectional converter considered in this paper is shown in Figure 1. The synchronised switches couple $Q_{1}-Q_{2}$ is controlled with an on/off signal. The converter is connected between two busses, a HV bus and a LV one, and acts as a bridge between the two busses, allowing the flow of power between them, in both directions. The HV side has a DC generator represented by an ideal voltage source $E_{H}$ and its internal resistance $R_{H}$, and also the battery on the LV side is modelled in the same way, with $E_{L}$ and $R_{L}$. Since in this paper no reactive power is considered, the unknown loads are modelled by a simple resistor $R_{D}$ whose value is supposed to be uncertain but limited and different from zero. By reducing the value of $R_{D}$ the connection of more loads (and the request for more power) is modelled. Obviously a value $R_{D}=0$ would result into infinite power request, and for this reason it is ruled out.

The current supplied by the generator, $I_{g}$, is supposed to be able to reach safely a nominal value $I_{g n}$ for long time intervals, where $I_{g n}$ is related to the rated power $P_{\mathrm{OL}}$ discussed in Introduction, while exceeding $I_{g n}$ is allowed only for short time, related to the 5-second and 5-minute capability.

Let $u=1$ refer to the configuration $\left(Q_{1}\right.$ on, $Q_{2}$ off $)$ and $u=0$ to $\left(Q_{1}\right.$ off, $Q_{2}$ on $)$. Then the equations of the converters are

$$
\begin{aligned}
& \dot{x}_{1}=-\frac{1}{L} x_{3}+\frac{1}{L} x_{2} u, \\
& \dot{x}_{2}=-\frac{1}{C_{H}}\left(\frac{1}{R_{H}}+\frac{1}{R_{D}}\right) x_{2}-\frac{1}{C_{H}} x_{1} u+\frac{E_{H}}{R_{H} C_{H}}, \\
& \dot{x}_{3}=\frac{1}{C_{L}} x_{1}-\frac{1}{R_{L} C_{L}} x_{3}+\frac{1}{R_{L} C_{L}} E_{L}, \\
& y=x_{1}
\end{aligned}
$$

where $x_{1}$ is the current flowing through the inductor $L, x_{2}$ is the voltage on the capacitor $C_{H}$ on the high-voltage bus side, and $x_{3}$ is the voltage on the capacitor $C_{L}$ on the low-voltage bus side. 


\section{Control Design}

The battery is controlled by a two-layer control. The lower layer guarantees that the current in the inductor $L$ follows a prescribed current reference, while the higher layer is a supervisor to manage power operations.

3.1. Low-Level Control. The current controller is based on the definition of a sliding manifold where the system output is required to be confined. Define a sliding manifold

$$
\mathcal{S}=\left\{(y, t) \mid \sigma\left(t, y, x_{2}\right)=0, \forall t \geq 0\right\},
$$

where the sliding function $\sigma$ is

$$
\sigma\left(t, y, x_{2}\right)=k x_{2}-y-\eta(t)
$$

where $k$ is a scalar constant and $\eta(t)$ is a smooth function vanishing with time and such that $\sigma\left(0, y(0), x_{2}(0)\right)=0$. This in turn requires $\eta(0)=k x_{2}(0)-y(0)$. The selection of $\eta$ and the definition of the control is the objective of the following Theorem.

Theorem 1. Consider system (1)-(4) and the control law

$$
u(t)=\frac{1}{\epsilon}\left(\sigma\left(t, y, x_{2}\right)+\gamma \int_{0}^{t} \sigma\left(\tau, \mathrm{y}, x_{2}\right) d \tau\right)
$$

with $\gamma>0$ and $\epsilon$ "small" positive constant. Let moreover $\eta$ be such that

$$
\begin{aligned}
\epsilon \ddot{\eta}+\dot{\eta}+c \eta & =0, \\
\eta(0) & =k x_{2}(0)-y(0), \\
\dot{\eta}(0) & =0,
\end{aligned}
$$

where $c>$ is a scalar positive constant. Assume $x_{2}(0)>0$. Then for $\epsilon \rightarrow 0$ the closed-loop system has one exponentially stable steady-state solution and there exists a $T=T(\epsilon)>0$ such that

$$
\left|y-k x_{2}\right|<\delta+\alpha e^{-c t}, \quad \forall t>T,
$$

where $\alpha$ and $\delta$ are suitable positive constants.

Proof. Preliminarily, consider the Lyapunov function

$$
V(x)=\frac{1}{2}\left(L x_{1}^{2}+C_{H} x_{2}^{2}+C_{L} x_{3}^{2}\right)
$$

and compute its time derivative along the trajectory of (1)-(3)

$$
\dot{V}(x)=-\left(\frac{1}{R_{H}}+\frac{1}{R_{D}}\right) x_{2}^{2}-\frac{1}{R_{L}} x_{3}^{2}+\frac{E_{H}}{R_{H}} x_{2}+\frac{E_{L}}{R_{L}} x_{3} ;
$$

thus, $\dot{V} \leq 0$ for $x$ outside a ball of a suitable radius; that is, the trajectories can not diverge. Moreover, the control law (7) is the solution of the differential equation

$$
\epsilon \dot{u}=\dot{\sigma}+\gamma \sigma \text {. }
$$

Then, the set of differential equations (1)-(4), (8), (12) can be studied resorting to the Theory of Singular Perturbations [20], with $\dot{\eta}$ and $u$ being "fast" variables, while $x_{i}, i=1, \ldots, 3$ and $\eta$ are "slow." Assumption $x_{2}(0)>0$ is necessary and sufficient for the stability of the fast subsystem. Letting $\epsilon=0$ one has $\dot{\sigma}_{0}+\gamma \sigma_{0}=0, \dot{\eta}_{0}+c \eta_{0}=0$, where the subscript " 0 " refers to the solution of the system with $\epsilon=0$. Note that since $\sigma\left(0 y(0), x_{2}(0)\right)=0$, then $\sigma=0$ for all times. In order to prove the stability of the slow subsystem, let $\epsilon=0$ in (12) to compute the equivalent control

$$
\begin{aligned}
u_{\mathrm{eq}} & =\frac{1}{\left(L k^{2}+C_{H}\right) x_{20}-L k \eta_{0}}\left[-k L\left(\frac{1}{R_{H}}+\frac{1}{R_{D}}\right) x_{20}\right. \\
& \left.+C_{H} x_{30}+\frac{E_{H} L k}{R_{H}}+c C_{H} L \eta_{0}\right] .
\end{aligned}
$$

Note that $\eta_{0}$ satisfies

$$
\eta_{0}(t)=e^{-c t} \eta_{0}(0)
$$

Then, since the trajectories of (1)-(3) can not diverge, the control after some time depending on the positive constant $c$ can be approximated as

$$
\begin{gathered}
u_{\text {app }}=\frac{1}{\left(L k^{2}+C_{H}\right) x_{20}}\left[-k L\left(\frac{1}{R_{H}}+\frac{1}{R_{D}}\right) x_{20}\right. \\
\left.+C_{H} x_{30}+\frac{E_{H} L k}{R_{H}}\right] .
\end{gathered}
$$

Substituting this control in (2) and (3) and taking into account $\sigma_{0}=0$ we obtain the reduced order system with solutions $x_{20}$ and $x_{30}$ satisfying

$$
\begin{aligned}
& \dot{x}_{20}=\frac{1}{L k^{2}+C_{H}}\left[-\left(\frac{1}{R_{D}}+\frac{1}{R_{H}}\right) x_{20}-k x_{30}+\frac{E_{H}}{R_{H}}\right], \\
& \dot{x}_{30}=\frac{1}{C_{L}} k x_{20}-\frac{1}{R_{L} C_{L}} x_{30}+\frac{E_{L}}{R_{L} C_{L}} .
\end{aligned}
$$

Since the whole reduced system has become linear, it is trivial to assess the global exponential stability of the slow subsystem (16) for any $k$. Thus, both the fast and the slow subsystems have been proved to be asymptotically stable, and (9) follows from Tikhonov's Theorem on the Infinite Time Horizon [21].

Theorem 1 proves the asymptotical convergence of $y$ to $k x_{2}$. However, in practical applications it is necessary to regulate $y$ to a prescribed constant reference $\bar{y}$, for example, when charging the battery with constant current. A possible solution is to compute a value of $k$ that guarantees a steadystate solution of $x_{2}$ so that the steady-state solution of $y$ is $\bar{y}$. This issue is addressed by the following Corollary.

Corollary 2. Consider system (1)-(4), with control (12), and consider a constant reference $\bar{y}$ such that

$$
\bar{y}<\frac{E_{H}^{2}}{4 R_{H} R_{L}\left(1+R_{H} / R_{D}\right)}-\frac{E_{L}}{R_{L}} .
$$


Select $k$ as one of the two values

$$
k=\frac{E_{H} \pm \sqrt{E_{H}^{2}-4 R_{H} \bar{y}\left(E_{L}+R_{L} \bar{y}\right)\left(1+R_{H} / R_{D}\right)}}{2 R_{H}\left(E_{L}+R_{L} \bar{y}\right)} .
$$

Then, the closed-loop system is such that

$$
\lim _{\epsilon \rightarrow 0} \lim _{t \rightarrow \infty} y(t)=\bar{y}
$$

Proof. The corollary follows by computing the equilibrium solution $\left(\bar{x}_{20}, \bar{x}_{30}\right)$ of (16) along with the equation $k \bar{x}_{20}=\bar{y}$. It results into a second-order algebraic equation, with real solutions if the discriminant is positive. Condition (17) is needed for the existence of real values for $k$.

The other objective of this paper is to limit the generator current at a maximum prescribed value. As it will be shown in the next section, this is equivalent to define a constant reference value for the voltage $x_{2}$. Thus a tool for tracking a constant reference on the state variable $x_{2}$ is needed. Also in this case, we will compute a $k$ that guarantees that the required $x_{2}$ is reached at steady-state. The solution is given by the following corollary.

Corollary 3. Consider system (1)-(4), with control (12), and consider a constant reference $\bar{x}_{2}$ such that

$$
\bar{x}_{2}<\frac{1}{2}\left(\frac{\rho}{R_{H}} E_{H}+\sqrt{\left(\frac{\rho}{R_{H}} E_{H}\right)^{2}+\frac{\rho}{R_{L}} E_{L}^{2}}\right) \text {, }
$$

where $1 / \rho=1 / R_{D}+1 / R_{H}$, and select $k$ as one of the two values

$k$

$$
=\frac{-E_{L} \pm \sqrt{E_{L}^{2}-4 R_{L} \bar{x}_{2} / R_{H}\left[\left(1+R_{H} / R_{D}\right) \bar{x}_{2}-E_{H}\right]}}{2 R_{L} \bar{x}_{2}} .
$$

Then, the closed-loop system is such that

$$
\lim _{\epsilon \rightarrow 0} \lim _{t \rightarrow \infty} x_{2}(t)=\bar{x}_{2} .
$$

Proof. Also this corollary easily follows by computing the equilibrium solution of (16) with $k \bar{x}_{2}=\bar{y}$ and imposing $\bar{x}_{20}=\bar{x}_{2}$.

Remark 4. In both Corollaries 2 and 3 two values for $k$ are possible, resulting in the same steady-state value for the controlled variable. The selection of the value is applicationdependent, based on the following considerations.

In the case of Corollary 2, since the current $\bar{y}$ is fixed, the higher the value for $k$ the lower the value of $x_{2}$. Thus, high values of $k$ result in $\mathrm{HV}$ voltage to drop from their nominal value. In the case of Corollary 3, on the contrary, $x_{2}$ is prescribed; hence a high value for $k$ results into large current in the inductor.

In the simulations presented in Section 4 we have always selected the lower value (in absolute value) for $k$.
Remark 5. The control law (7) presented so far is continuous, while the switching converter requires a control variable $u$ that has only binary values; that is, it only assumes value 0 or 1 . Thus, the control strategy in Theorem 1 must be realised with a Heaviside function replacing the high gain $1 / \epsilon$ or with $\Sigma-\Delta$ modulator [15] to be implemented on the switching converter. It is possible to show as in [22] that in order to correctly implement the proposed strategy with a binary control, the condition $0 \leq u_{\mathrm{eq}} \leq 1$ must hold. Note that this implementation is exact, that is, without approximations.

Remark 6. The exponential term $\eta$ has the effect of making an initial modification of the trajectory reference, so that the system output starts from the sliding surface from the beginning. However, this implies to reach the reference exponentially. If this is not acceptable, it is possible to reach the reference $\bar{y}$ in finite time by exploiting finite-time convergent controllers [23]. For instance, it is possible to show that the control law

$$
u(t)=H_{\delta}\left(\tilde{\sigma}(y)+\gamma_{1} \operatorname{sign} \widetilde{\sigma} \int_{0}^{t} \sqrt{|\widetilde{\sigma}(y)|} d \tau\right),
$$

where $\widetilde{\sigma}=\bar{y}-y$ and $H_{\delta}(\cdot)$ is relay function with values 0 and 1 and hysteresis band $\pm \delta$, steers the output $y$ to the desired output $\bar{y}$ in finite time. Note that in this case expression (15) is the exact equivalent control, not its approximation.

Theorem 1, used jointly with the two corollaries, enables the control designer to track current references on the inductor or voltage references on the HV side capacitor. This capability will be exploited in the next section to define a supervisory control strategy for the bidirectional BBCU.

3.2. High-Level Control. The high-level controller is devoted to smart management of the electric energy onboard. Usually, when batteries or other energy rechargeable storage devices are used a controller looking at the state of charge of the device is used. In this paper we will neglect this aspect to concentrate strictly on the action of helping the generator to supply the loads. In fact, we will assume the following, reasonable basic hypothesis:

Hypothesis 7. For each time instant, the total load energy demand does not exceed the sum of the energy supplied by the generator until the time instant and the energy stored in the battery onboard.

As a consequence of the above hypothesis, we are assuming that the loads require extra power for limited time, because it is clear that if the load requires extra power for long time, the basic assumption would be violated and a larger generator would be required. Note that this does not mean that the battery is not charged. On the contrary, the battery is recharged at constant current when possible, that is, when the extra energy demand ends. This explains why we can neglect the state of charge requirement. Note that the constant current charge can be obtained by exploiting Corollary 2. Extra energy to the loads implies that the generator is supplying its maximum 5-minute current, while 
the battery supplies the extra current by using Corollary 3 to compute the value of $k$ needed for the control. Preliminarily, a reference on the HV side capacitor voltage must be defined. This is done by simply noticing that the current from the generator can be expressed as

$$
I_{g}=\frac{E_{H}-x_{2}}{R_{H}} .
$$

Since, as noticed in Section 2, the rated generator current $I_{g n}$ is known in advance, from (24) a desired value for $\bar{x}_{2}$ is derived.

Note that also condition (20) must be translated in terms of $I_{g n}$, resulting in

$$
I_{g n}>\left(1-\frac{1}{2} \phi_{H}\right) i_{H}-\frac{1}{2} \sqrt{\phi_{H}^{2} i_{H}^{2}-\phi_{L} i_{L}^{2}},
$$

where $\phi_{j}=\rho / R_{j}$ and $i_{j}=E_{j} / R_{H}, j \in\{H, L\}$, with $\rho$ defined in Corollary 3. that is,

In conclusion, the high-level supervisor has two states,

\section{(1) constant inductor current (state $S 1$ );}

(2) constant generator current (state S2).

The commutation between the two modes is given by the event of current overload on the generator. Moreover, in order to avoid chattering between the two phases, the commutation happens with hysteresis with band $\left[I_{g n}-\theta, I_{g n}+\theta\right]$, rather than a strict threshold.

\section{Simulation Results}

The control structure presented so far has been tested on a detailed simulator designed in the Matlab environment, using the SimPowerSystems package of the Simscape Library. It allows considering detailed switching elements with different parasitic effects, snubber resistance and capacity, and so on, so as to obtain very accurate simulations. Moreover, also a realistic model of the battery has been considered. The complete simulation scheme is shown in Figure 2. The HV grid has parameters $E_{H}=270 \mathrm{~V}$ and $R_{H}=100 \mathrm{~m} \Omega$. The "Intelligent Battery," shown in the green block in Figure 3, is comprised of

(i) battery (the detailed model available in the library has been used);

(ii) supervisor;

(iii) controller;

(iv) interface with logic switches.

The parameters of the battery are nominal voltage $28 \mathrm{~V}$, rated capacity $9 \mathrm{Ah}$, and internal resistance $R_{L}=47.5 \mathrm{~m} \Omega$. The converter has $L=10 \mathrm{mH}, C_{H}=800 \mu \mathrm{F}$, and $C_{L}=400 \mu \mathrm{F}$. The controller parameters are $\gamma=1, \epsilon=0.1, \theta=0.3$, and $I_{g n}=16 \mathrm{~A}$. The variable load $R_{D}$ is represented by a block containing five resistances with the following values: $R_{D 1}=300 \Omega, R_{D 2}=200 \Omega, R_{D 3}=100 \Omega, R_{D 4}=16.8 \Omega$, and
$R_{D 5}=15 \Omega$. The resistors are individually connected, in order to simulate variable loads. The supervisor is implemented in State Flow as depicted in Figure 4.

The generator current, needed only to detect the event of current overload, is estimated through the low-pass filter

$$
\begin{aligned}
& \dot{x}=-\frac{1}{\tau_{g}} x+\frac{1}{\tau_{g}} I_{g}, \\
& y=x
\end{aligned}
$$

with $\tau_{g}=0.01 \mathrm{~s}$, to smooth high-frequency noise due to switching implementation.

At the beginning of the simulation, the supervisor is in state $S 1$ with load $R_{D}=R_{D 1}$, so the goal is to drive $x_{1}$ to $\bar{y}=3.6 \mathrm{~A}$, that is, $40 \%$ the rated capacity of the battery, as in standard practice. The battery is assumed to start from $80 \%$ its maximum charge. Figure 5 shows that the current starts from initial condition $x_{1}(0)=1 \mathrm{~mA}$ and quickly reaches the reference. At $t=5 \mathrm{~s}$ and $t=10 \mathrm{~s}$ the load changes first to $R_{D 2}$ and then to $R_{D 3}$. The generator current is automatically increased, but it is much below the overload current, and so the supervisor remains in state $S 1$. At time instant $t=15 \mathrm{~s}$ a new load is added, resulting in a reduction of total load resistance to $R_{D}=R_{D 4}$. In this case the overload capacity of the generator is exceeded, so the supervisor switches to state $S 2$, and it changes the objective of the control. The new objective is to keep $I_{g}$ to $I_{g n}$, as Figure 6 shows. Note that the 5-second capability of the generator is not exceeded. Interestingly, the generator is still able to charge the battery, although with a small positive current. This is a characteristic advantage of the proposed controller; the generator charges the battery as long as possible. Next, at $t=20 \mathrm{~s}$ the load changes again to $R_{D 5}$. Now the generator is no longer able to supply the battery, and the inductor current changes sign; that is, the battery helps the generator to supply the loads. Finally the load returns to initial condition of $300 \Omega$, and the controller automatically recovers its initial state. As mentioned in the previous section, a complete battery management system should consider also the state of charge of the battery. However, since only transient loads are considered in this paper, the low-battery event is not presented here. Figure 8 shows the state of charge of the battery during the simulation.

Also the LV side capacitor voltage is reported, in Figure 7. In this case some considerations about the flow of energy can be drawn, confirming the above considerations. Indeed, after the initial transient, when the capacitor voltage starts from $x_{3}(0)=25 \mathrm{~V}$, the controlled capacitor voltage always exceeds $E_{L}=28 \mathrm{~V}$ so that the flow of energy is towards the battery. Only after time $t=20 \mathrm{~s}$ the flow of energy is reversed, as the voltage drops below $28 \mathrm{~V}$.

Moreover, the condition in Remark 5 is checked, by plotting the equivalent control in Figure 9. It is clear that the equivalent control is well within the interval $[0,1]$.

Finally, the finite-time implementation proposed in Remark 6 is tested. The results shown in Figures 10 and 11 for the inductor and generator current, respectively, show that the finite-time controller implementation is preferred when shorter transients in the generator are needed and quick 


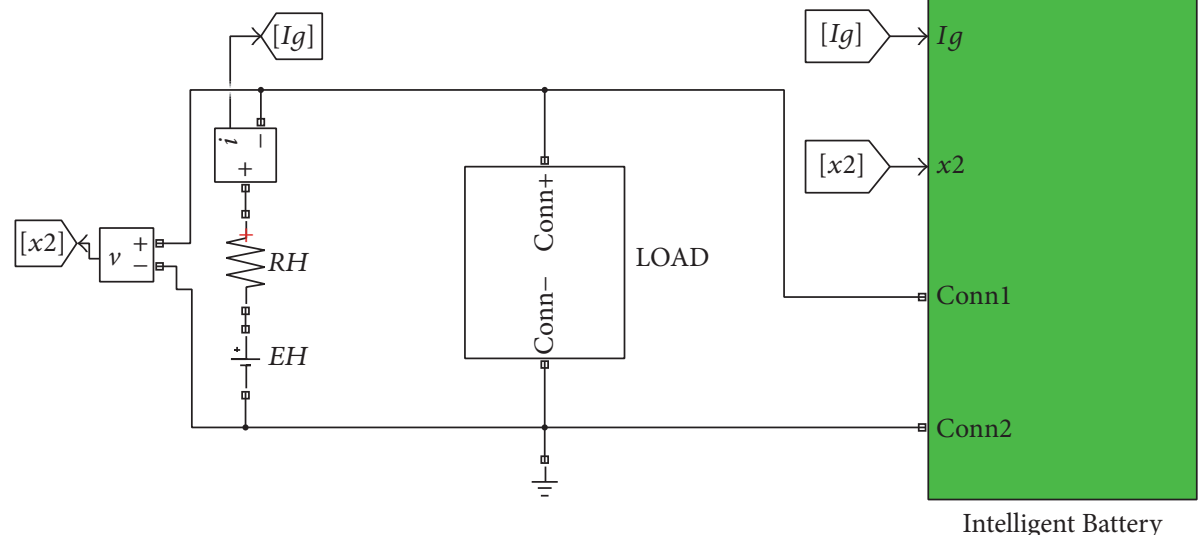

FIgURE 2: Complete simulation scheme.

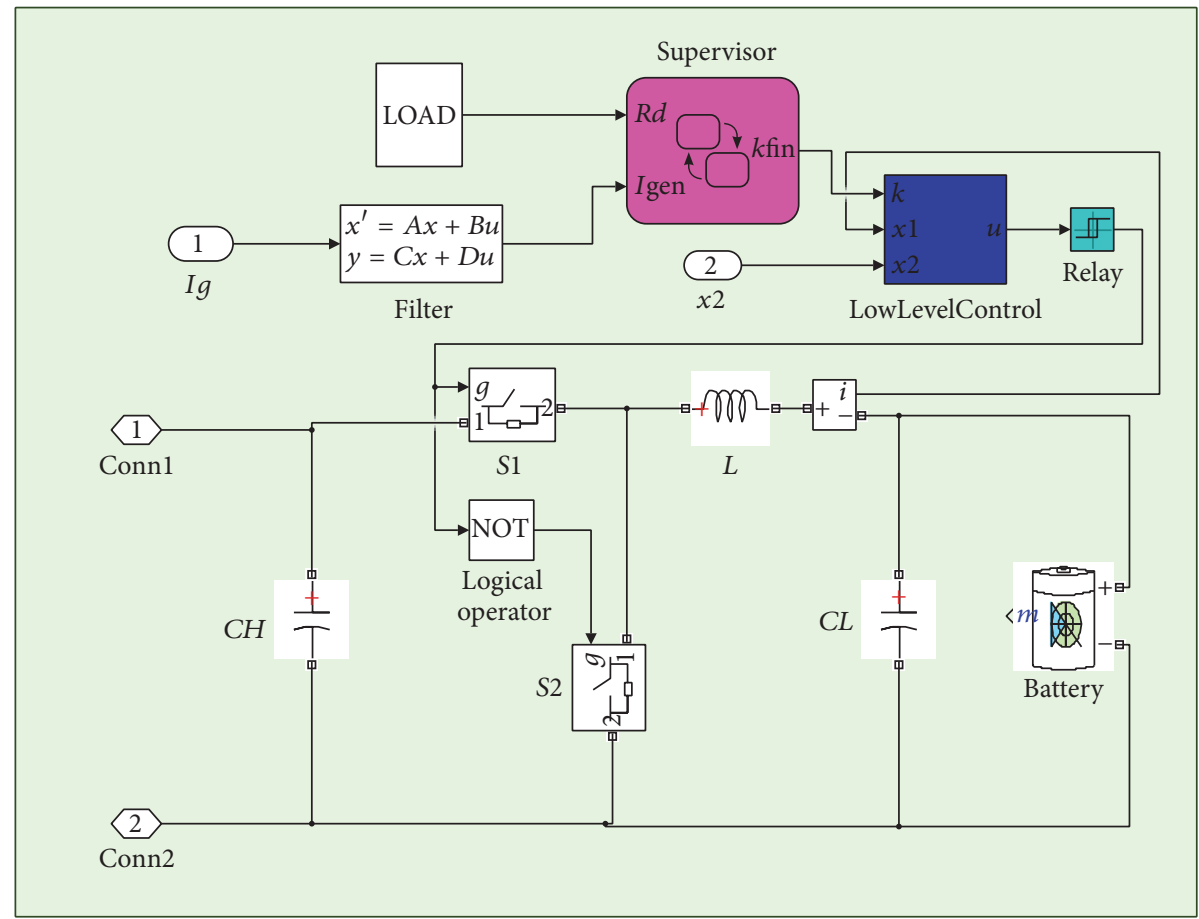

Figure 3: Intelligent Battery Module.

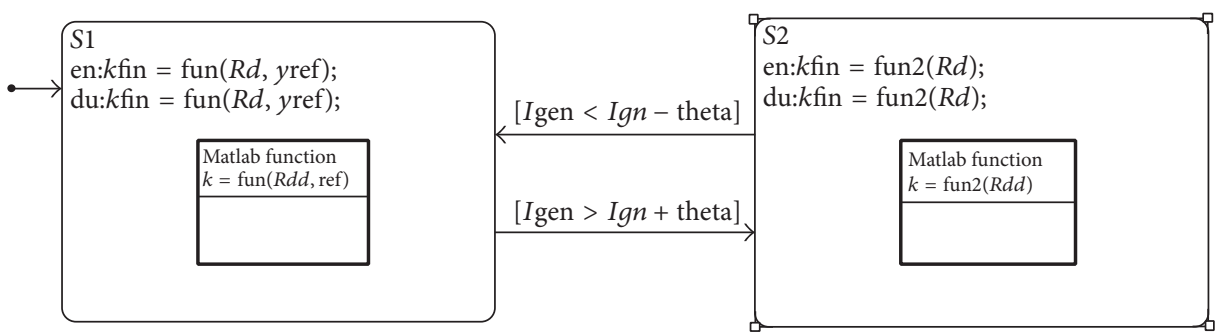

Figure 4: Supervisor. 


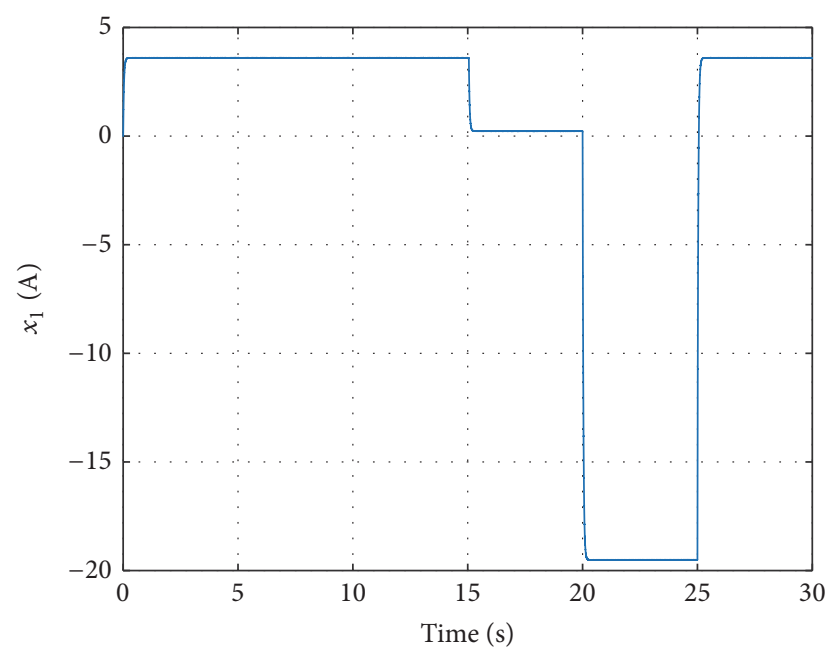

Figure 5: Inductor current.

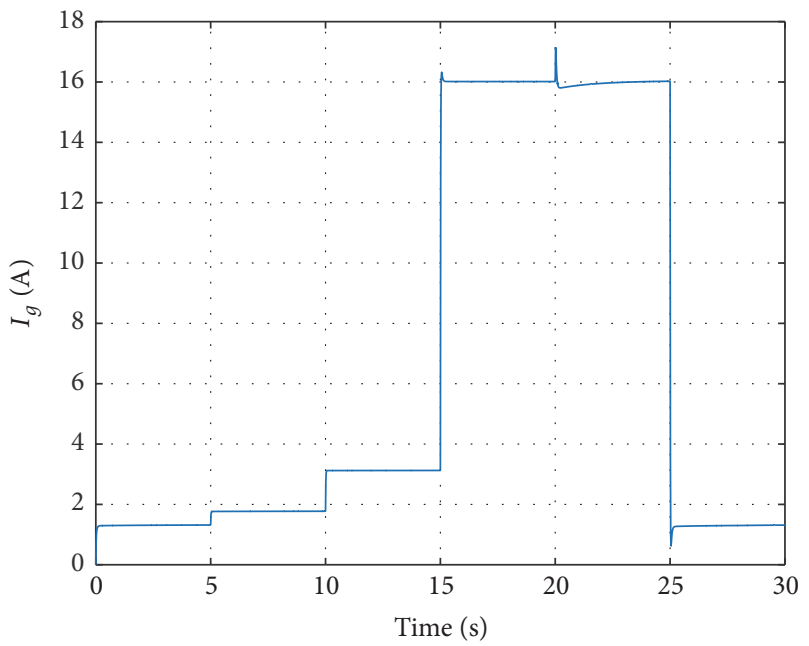

FIGURE 6: Generator current.

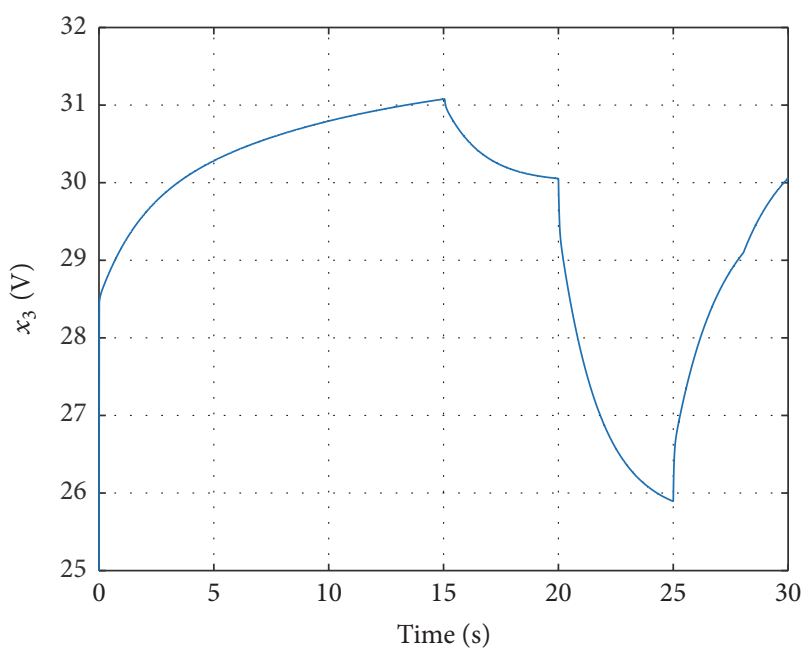

FiguRe 7: LV bus voltage.

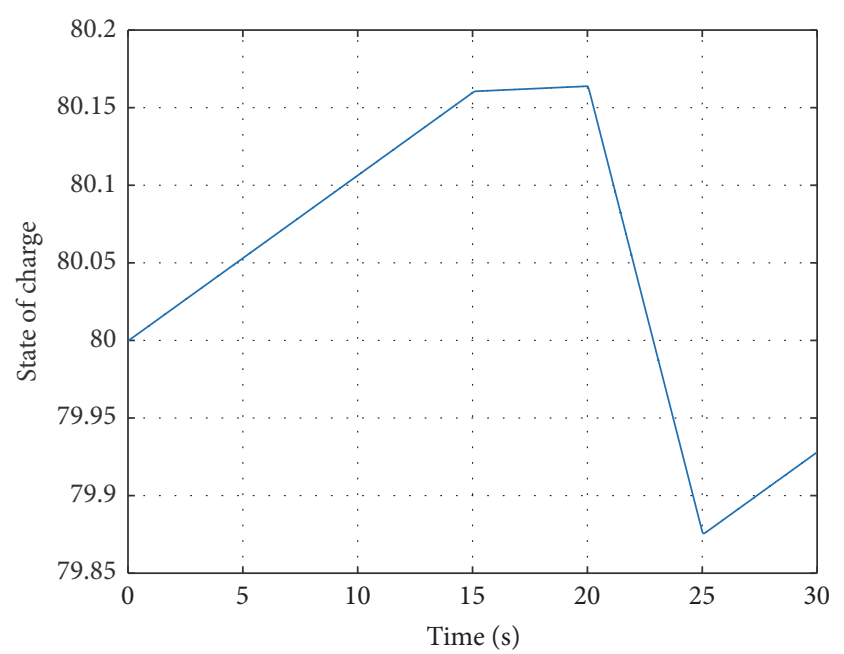

FiguRE 8: State of charge of the battery.

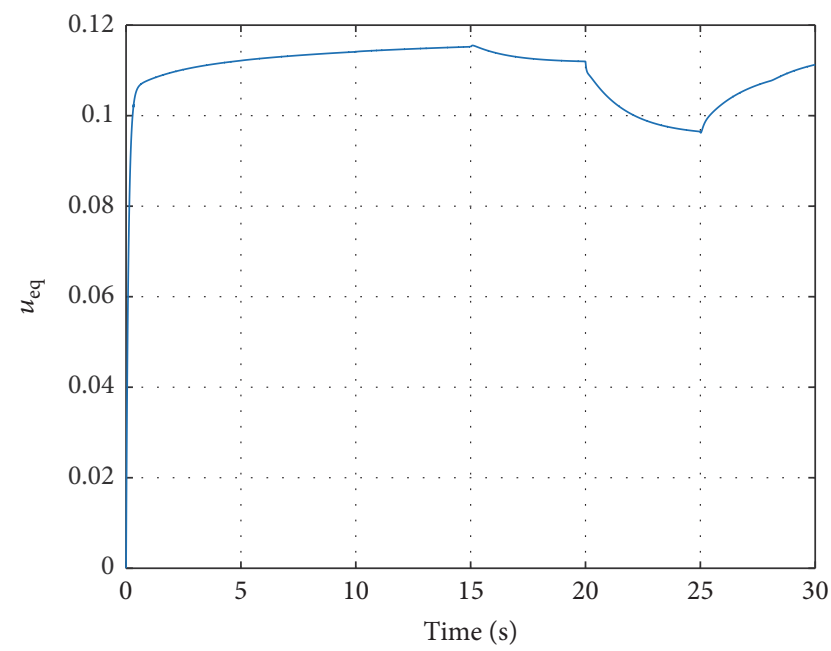

Figure 9: Equivalent control.

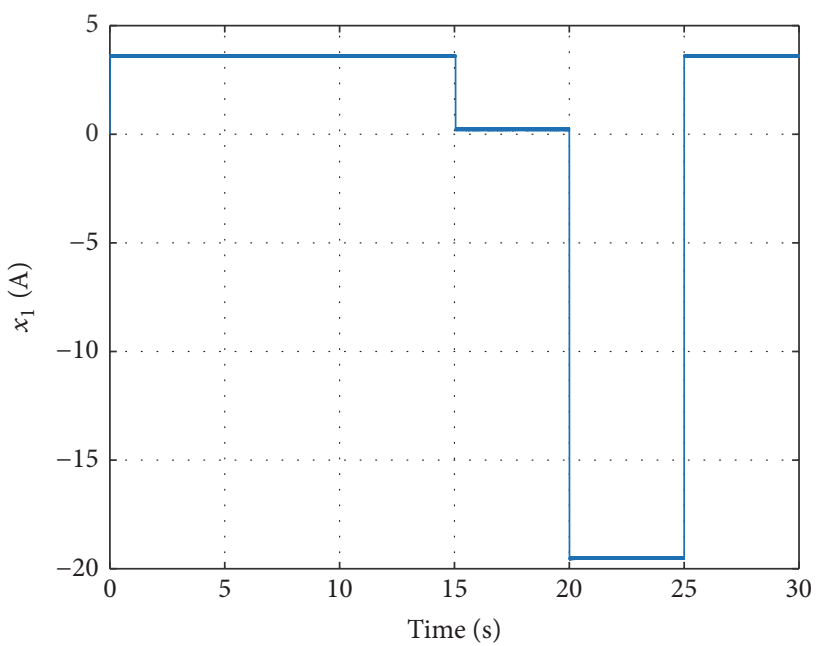

Figure 10: Inductor current (finite-time controller). 


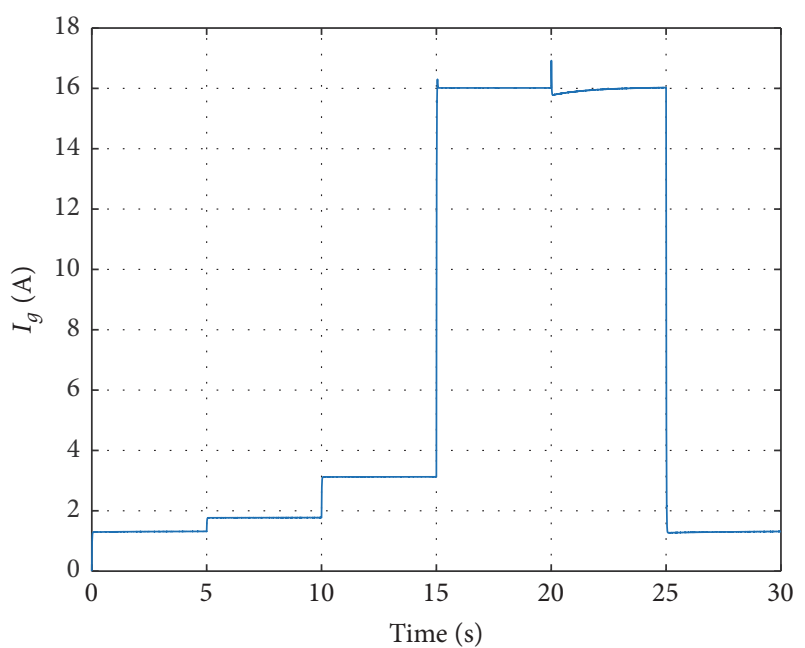

FIGURE 11: Generator current (finite-time controller).

transient in the energy storage device are acceptable, as is the case when the battery is replaced by a quicker supercapacitor (as in [24]).

\section{Conclusions}

In this paper a strategy for the control of a battery for aeronautic applications has been presented. The objective is to use the battery as a "power buffer" that helps the power generator of the aircraft when extra current is required from loads on the high-voltage DC bus. The solution proposed in this paper is based on a supervisory controller that schedules the action of two low-level controllers. The low-level controllers, in turn, have the objective of keeping the current to the battery and the current drawn from the generator, respectively, constant. Also a finite-time control implementation of both strategies is considered and discussed. An automaton implementation is used for the supervisor and a formal proof of stability with different loads is discussed. Detailed simulations with Matlab, Simulink, and SimPowerSystem blockset are performed, considering also a realistic implementation of the switching elements and of the battery. A complex simulation scenario encompassing different loads varying during time is presented to show the effectiveness and the robustness of the proposed strategy.

\section{Conflicts of Interest}

The authors declare that there are no conflicts of interest regarding the publication of this paper.

\section{Acknowledgments}

This research is being conducted in the frame of Cleansky JTI project (EPOCAL no. 323408), an FP7 European Integrated project, http://www.cleansky.eu.

\section{References}

[1] A. Emadi and M. Ehsani, "Aircraft power systems: technology, state of the art, and future trends," IEEE Aerospace and Electronic Systems Magazine, vol. 15, no. 1, pp. 28-32, 2000.

[2] A. Cavallo, C. May, A. Minardo, C. Natale, P. Pagliarulo, and S. Pirozzi, "Active vibration control by a smart auxiliary mass damper equipped with a fiber Bragg grating sensor," Sensors and Actuators, A: Physical, vol. 153, no. 2, pp. 180-186, 2009.

[3] A. Cavallo, C. Natale, S. Pirozzi, and C. Visone, "Limit cycles in control systems employing smart actuators with hysteresis," IEEE/ASME Transactions on Mechatronics, vol.10, no. 2, pp.172180, 2005.

[4] D. Schlabe and J. Lienig, "Energy management of aircraft electrical systems-state of the art and further directions," in Proceedings of the Electrical Systems for Aircraft, Railway and Ship Propulsion (ESARS '12), pp. 1-6, October 2012.

[5] POA, “(2002-2005) Poa-power optimised aircraft project," http://www.2020-horizon.com/POA-Power-optimised-aircraft(POA)-s16028.html.

[6] L. Faleiro, "Summary of the European Power Optimised Aircraft (POA) project," in Proceedings of the 25th Congress of the International Council of the Aeronautical Sciences 2006.

[7] MOET, (2006-2009) Moet-more open electrical technologies, http://cordis.europa.eu/project/rcn/81472_en.html.

[8] T. Jomier, Moet Project: Background \& Objectives, 2011, http:// www.cdti.es/recursos/doc/eventosCDTI/Aerodays2011/7B1.pdf.

[9] CleanSky, "(2010-2015) Clean sky website," http://www.cleansky.eu/innovative-technologies.

[10] S. Ghosh, Electrical Machines, Pearson Education, 2nd edition, 2012.

[11] B. Guida and A. Cavallo, "A Petri net application for energy management in aeronautical networks," in Proceedings of the IEEE 18th International Conference on Emerging Technologies and Factory Automation (ETFA '13), September 2013.

[12] L. Rubino, B. Guida, F. Liccardo, P. Marino, and A. Cavallo, "Buck-boost DC/DC converter for aeronautical applications," in Proceedings of the IEEE International Symposium on Industrial Electronics (ISIE '10), pp. 2690-2695, July 2010.

[13] B. Guida and A. Cavallo, "Supervised bidirectional DC/DC converter for intelligent fuel cell vehicles energy management," in Proceedings of the IEEE International Electric Vehicle Conference (IEVC '12), March 2012.

[14] L. Martinez-Salamero, A. Cid-Pastor, R. Giral, J. Calvente, and V. Utkin, "Why is sliding mode control methodology needed for power converters?" in Proceedings of the 14th International Power Electronics and Motion Control Conference, vol. 9, pp. 2531, September 2010.

[15] H. Sira-Ramirez and R. Silva-Ortigoza, Control Design Techniques in Power Electronic Devices, Power Systems, Springer, Berlin, Germany, 2006.

[16] V. Utkin, J. Guldner, and J. Shi, Sliding Mode Control in ElectroMechanical Systems, CRC Press, 2009.

[17] A. Cavallo and B. Guida, "Sliding mode control for DC/DC converters," in Proceedings of the 51st Annual Conference on Decision and Control (CDC '12), pp. 7088-7094, December 2012.

[18] A. Cavallo, B. Guida, A. Buonanno, and E. Sparaco, "Smart Buck-Boost Converter Unit operations for aeronautical applications," in Proceedings of the 54th IEEE Conference on Decision and Control (CDC '15), pp. 4734-4739, December 2015. 
[19] S. Ding, A. Levant, and S. Li, "New families of high-order sliding-mode controllers," in Proceedings of the 54th IEEE Conference on Decision and Control (CDC '15), pp. 4752-4757, December 2015.

[20] H. Khalil, Nonlinear Systems, Prentice Hall, 3rd edition, 2002.

[21] F. C. Hoppensteadt, "Singular perturbations on the infinite interval," Transactions of the American Mathematical Society, vol. 123, pp. 521-535, 1966.

[22] H. Sira-Ramirez, "Sliding motions in bilinear switched networks," IEEE Transactions on Circuits and Systems, vol. 34, no. 8, pp. 919-933, 1987.

[23] A. Levant, "Universal single-input-single-output (SISO) sliding-mode controllers with finite-time convergence," IEEE Transactions on Automatic Control, vol. 46, no. 9, pp. 1447-1451, 2001.

[24] A. Buonanno, E. Sparaco, A. Cavallo et al., "Rate-limiter control comparison for energy storage systems in aerospace applications," in Proceedings of the 8th International Conference on Power Electronics, Machines and Drives, April 2016. 


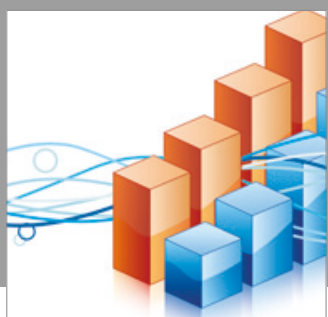

Advances in

Operations Research

vatersals

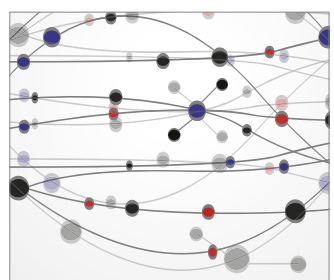

\section{The Scientific} World Journal
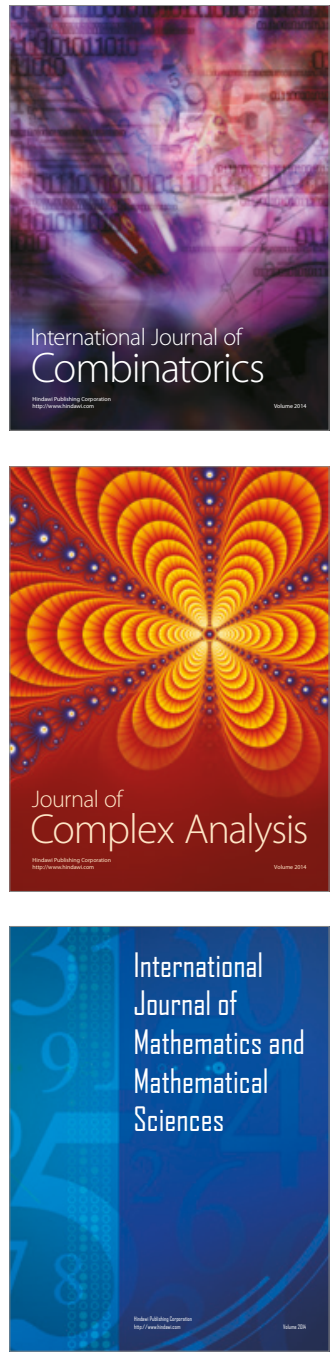
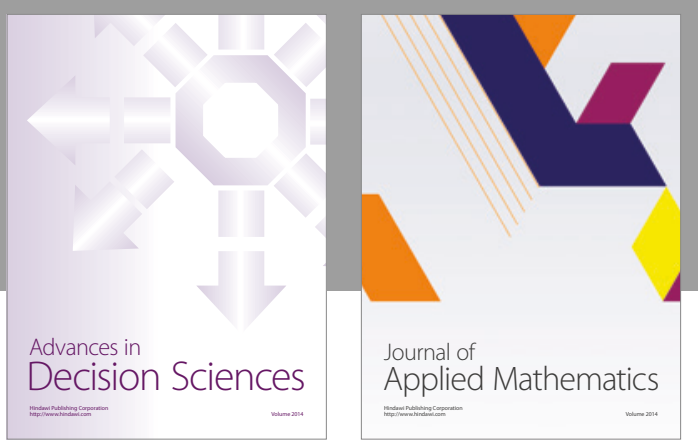

Algebra

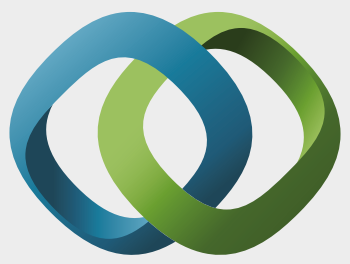

\section{Hindawi}

Submit your manuscripts at

https://www.hindawi.com
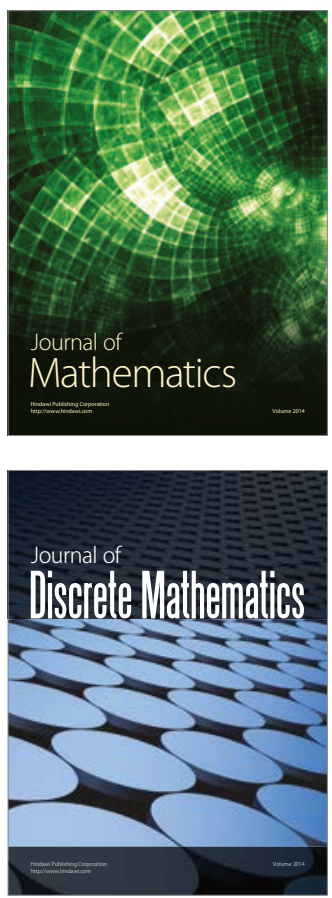

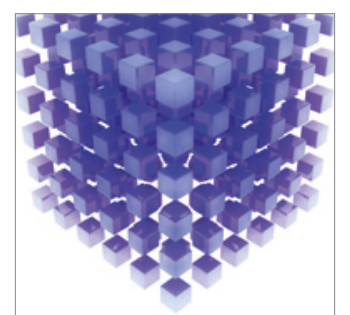

Mathematical Problems in Engineering
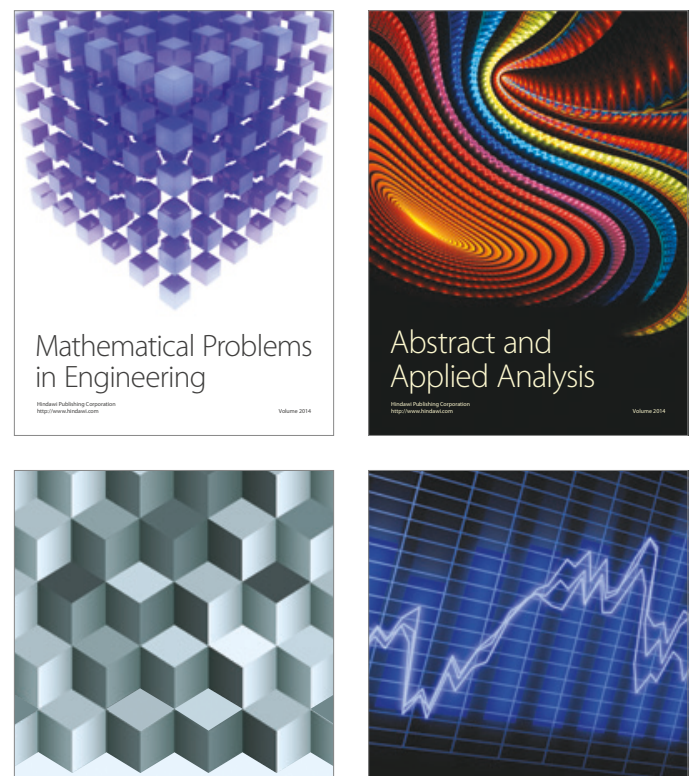

Journal of

Function Spaces

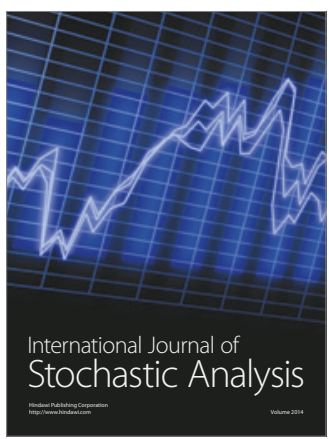

Probability and Statistics
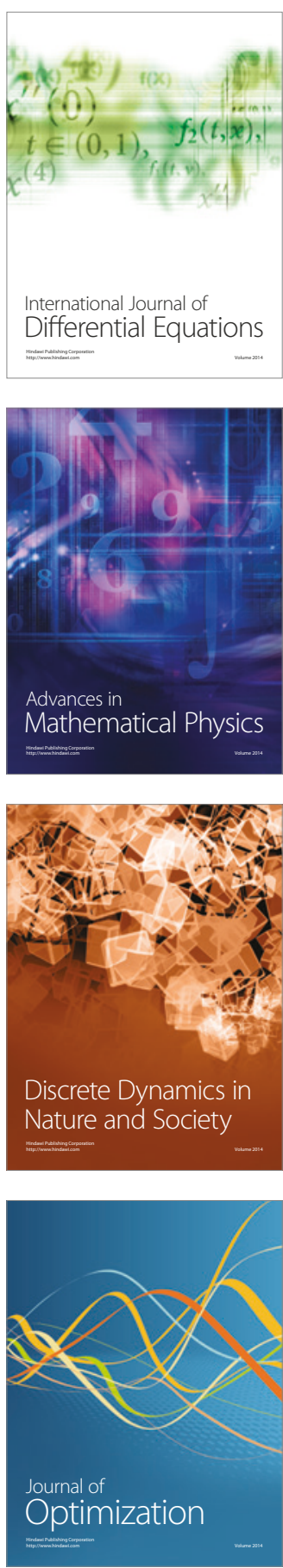